\title{
Pediatric Assent for a Study of Antiretroviral Therapy Dosing for Children in Western Kenya: A Case Study in International Research Collaboration
}

RACHEL C. VREEMAN Indiana University School of Medicine

Winstone M. NyAndiko

Moi University School of Medicine

ERIC M. MesLin

Indiana University Center for Bioethics

ABSTRACT: MULTINATIONAL COLLABORATORS IN health research face particular ethical challenges when conducting studies involving vulnerable populations such as children. We use an example from our first attempt to implement pediatric assent in the context of a longstanding research and clinical partnership between Kenyan and American medical schools to highlight the ethical and procedural issues related to pediatric assent that must be considered for multinational, pediatric studies. We consider relevant domestic, professional, and international guidelines for assent in pediatric research subjects, and we discuss the particular ethical challenges related to pediatric assent in the Kenyan context. Finally, we propose a way forward for approaching pediatric assent within our collaborative research program in Kenya that may apply to other multinational research partnerships.

KEY WORDS: assent, pediatric assent, children

Received: January 7, 2009; revised February 1, 2009

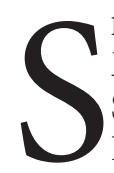

INCE 2001, THE INDIANA UNIVERSITY SCHOOL of Medicine has partnered with the Moi University School of Medicine, one of three medical schools in Kenya, to provide care for over 85,000 adults and children in Western Kenya who are infected with Human Immunodeficiency Virus (HIV). This collaboration, recently renamed the USAID-Academic Model Providing Access To Healthcare (AMPATH) Partnership, has a tripartite mission to provide clinical care, to enhance medical education, and to conduct research. A key component of this collaboration has been attention to the way in which ethical issues are addressed in jointly conducted research. One of us (EMM) reported previously in this journal of our efforts to better understand the ethics needs of investigators and ethics review committees at Indiana University and at Moi University (Sidle et al., 2006). In this paper, we focus on a specific topic in international research ethics: the involvement of children.

Multinational research in developing countries can yield a number of ethical challenges as researchers attempt to avoid exploitation and to build true collaborative partnership (Emanuel et al., 2004). While informed consent is widely accepted as an ethical and legal requirement of clinical research, cultural and language differences add an additional level of difficulty (Emanuel et al., 2004). Obtaining informed consent for research involving children raises special, additional concerns, as children are generally not considered capable of informed consent. Research within this vulnerable population thus requires adults to provide consent for the children and prompts consideration of additional measures, such as obtaining children's assent. In this paper, we seek to describe and analyze particular ethical issues related to implementing pediatric assent policies within clinical research in western Kenya. While these challenges are of immediate relevance to the AMPATH clinical care system and research network, we also seek to illuminate the issues that may arise for conducting pediatric research in other resource-limited settings.

\section{A Case Study from Western Kenya}

Children infected with Human Immunodeficiency Virus (HIV) require antiretroviral therapy (ART) in order to stay alive and to fight their HIV infection. Through the AMPATH system of clinics in rural and urban Western Kenya, over 3,300 HIV-infected children currently receive free ART (Figure 1). Despite the widespread, international use of ART, the proper dosing of these medicines in children has not been well studied (Havens \& Gibb, 2007). Moreover, because $90 \%$ of

Journal of Empirical Research on Human Research Ethics, PP. 3-16. PRINT ISSN 1556-2646, ONLINE ISSN 1556-2654. (C) 2009 BY JOAN SIEBER. ALL RIGHTS RESERVED. PLEASE DIRECT ALL REQUESTS FOR PERMISSIONS TO PHOTOCOPY OR REPRODUCE ARTICLE CONTENT THROUGH THE UNIVERSITY OF CALIFORNIA PRESS'S RIGHTS AND PERMISSIONS WEBSITE, HTTP://WWW.UCPRESSJOURNALS.COM/REPRINTINFO.ASP. DOI: $10.1525 /$ jer.2009.4.1.3 


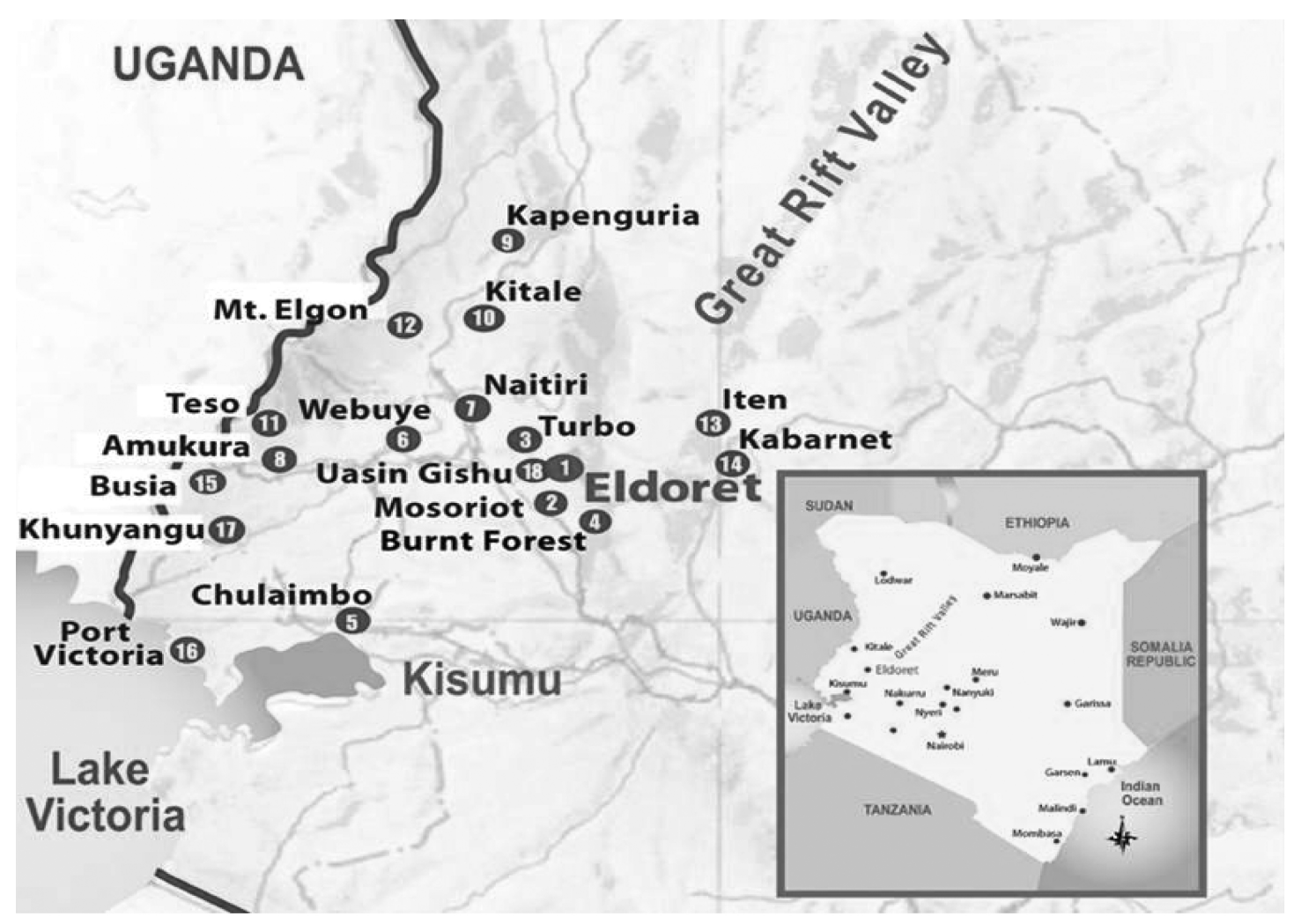

FIG. 1. Locations of the AMPATH HIV Care System in Western Kenya.

HIV-infected children live in Africa (UNICEF, 2007), many in resource-limited settings where children are more likely to be malnourished, it is especially important to find out whether the drug doses need to be adjusted for children who are malnourished. To address this knowledge gap, AMPATH pediatricians designed a study protocol to measure the pharmacokinetics or metabolism of nevirapine, one first-line antiretroviral medication for HIV-infected pediatric patients. In this study protocol, researchers proposed to evaluate both the nevirapine pharmacokinetics and the nutritional status of HIV-infected children ages three to thirteen years, who were initiating antiretroviral therapy with nevirapine. The objective of this research was to provide a pharmacokinetics model for nevirapine in children that could guide pediatric dosing for children in subSaharan Africa, with attention to how this dosing might need to change in the setting of malnutrition.

The pediatric nevirapine pharmacokinetics study required the children to stay overnight in the hospital on two occasions. During these two hospitalizations, the children would have five small blood samples drawn, from which the researchers would measure the level of the drug in the child's blood, as well as the child's nutritional status. This protocol was the most invasive research investigation proposed to date for children within the AMPATH research collaboration. For example, no previous prospective research studies of children within AMPATH required overnight hospitalization or even the drawing of blood exclusively for the purposes of research.

\section{Key Issues Arising from the Case}

As with most international collaborations between investigators located in different countries, it is customary (and in some countries required) that protocols be reviewed and approved by ethics review bodies in each country. Within the IU-Kenya research collaboration, study protocols must be reviewed and approved by both the local IRB in Indianapolis at Indiana University-Purdue University Indianapolis (IUPUI IRB) and the local Kenyan Institutional Research Ethics Committee of the Moi University School of Medicine (MU IREC). The requirement for joint review has been part of the standard operating procedures of these committees, both of which have federal-wide assurances on file with the U.S. Office for Human Research Protection and as such are 
required to be familiar with (and to comply with) the U.S. federal policy for the protection of human subjects (also called the "Common Rule," found at 45 CFR 46, Subpart A). Both review bodies are also familiar with the additional protections for research involving children (found at 45 CFR 46, Subpart D). We also note that nothing in these regulations stipulate how joint review will occur (e.g., whether review is done sequentially or simultaneously, whether one has veto power over the other, etc.). These are matters to be worked out on the ground (Sidle et al., 2006).

Given this brief background, we next describe the communication with each review committee, focusing more on the Kenyan communications, since these may be of more interest to readers less familiar with non-U.S. ethics review. We will then provide a more detailed assessment of what we learned.

\section{Communication with the Indianapolis Review Board}

The IUPUI IRB typically classifies protocols and what is required for consent or assent based on the risks to subjects compared to the potential benefits. By their published criteria, they considered the protocol of minimal risk to the pediatric subjects since the pediatric pharmacokinetics study required only a series of blood draws, but no other invasive procedures. Based on this degree of risk, the standard IUPUI institutional policy is to obtain written assent for children seven years of age and older. The national and international guidelines on which this policy is based are described in more detail later in the paper. In addition, the study participants had the potential to experience direct benefits in terms of drug dosing adjustments based on their individual characteristics and drug levels. These data would not be available in routine AMPATH clinical care. Since the investigators in the collaborative partnership had not previously conducted pediatric research of this kind in Kenya, one of the principal investigators queried members of the IUPUI IRB related to the issue of pediatric assent in this setting. In written communication prior to the submission of the full IRB protocol, the IUPUI IRB recommended that the protocol follow the standard IUPUI institutional policy for pediatric assent, where written assent is obtained for children seven years of age and older.

The AMPATH researchers working on this project agreed to try to introduce a requirement for pediatric assent as part of the protocol to investigate pediatric pharmacokinetics in Kenya. Factors influencing this original decision included the recommendations of the
IUPUI IRB, the desire to introduce specific protections for the vulnerable pediatric population in the Kenya research setting, and the specific features of the trialwhere direct benefit was possible, but not certain, and where the children would be required to undergo fairly burdensome procedures that included two nights in the hospital and a series of blood draws. The researchers designed the original study protocol to require that verbal, witnessed assent be obtained for children seven years of age and older.

This protocol was sent to the IUPUI IRB and MU IREC for review in tandem, and was reviewed by the IUPUI IRB first. The IUPUI IRB approved the protocol with no recommended revisions to the portion of the protocol involving pediatric assent or informed consent.

\section{Communication with the Eldoret Review Board}

The MU IREC approved the study, but communicated that much deliberation went into the approval of the protocol. There was no prior history of obtaining pediatric assent in research studies approved by MU IREC, so the recommendation of the IUPUI IRB presented a novel concept for consideration, and one that was met with some surprise and concern. Concerns were expressed about whether children under the age of fourteen had the right and the capacity to make autonomous decisions, with particular concern regarding the ability of children at this age to reason through the decisionmaking process necessary to consent. In some parts of Kenya, children are not viewed as autonomous decision makers and may have little experience with exercising autonomy. However, the view of the child, and also the child's ability to participate in this process, were thought to vary widely based on whether the child was from an urban or rural part of the country and the degree to which the child and parents had been educated (and, potentially, Westernized). Citing steadily changing and more progressive views of the Kenyan child's capacity and experiences, some thought that the society had reached a place where pediatric assent should be sought and valued.

Furthermore, there were logistic questions: In what language or languages would the assent procedures take place given that many children learn a tribal language as their primary language, followed by the more universal Kiswahili, and then may learn only English in school? Would written assent or verbal assent be used? For Kenyan adults, signing a document with words that you cannot read is considered inappropriate, even if the document has been read aloud in full (Sidle et al., 2006). 
Thus, previous studies in this setting have used the signatures of two witnesses to document verbal consent. Should a study in children employ the same techniques? Finally, would age criteria or some other criteria be used to determine for which children assent was required?

After considering these issues, the MU IREC approved a study protocol in which verbal, witnessed informed consent would be obtained from a parent or guardian of the participating child and that verbal, witnessed assent would be obtained for children seven years of age and older. Because this same protocol had previously received approval by the IUPUI IRB, the MU IREC discussion was not conveyed to the IUPUI IRB.

As we know, the mere outcomes of IRB review are a necessary but not a sufficient component for fully understanding overall research decision making in the international environment. Below we describe some of the key issues that help to explain some of the unique feature of these deliberations.

\section{Pediatric Assent in Multinational Research}

Our case revealed many important differences in both ethical and procedural issues in international research collaborations. The review committee from the sponsoring country (the IUPUI IRB) recommended following rules that written pediatric assent for children seven years of age and older must be obtained. The ethics review committee from the country in which the research would be conducted (MU IREC) had not previously implemented pediatric assent for children under fourteen years and questioned its appropriateness. As such, the protocol highlights raises a number of key questions facing all researchers in international collaborative studies: How should consensus be reached when clear consensus may not be present within a single country? When collaborating partners have different ideas about appropriate ethical standards-in this case, about the need to obtain pediatric assent from children over seven years of age-how should those differences be resolved?

To move toward answering these questions, we first consider the state of pediatric assent and its implementation in both settings. We then examine the international guidelines for consent and assent in pediatric research subjects. Next we discuss the particular ethical challenges related to the concept of pediatric assent, and then the challenges for pediatric assent specific to the Kenyan context. Finally, working from the consideration of the guidelines and the nuances of the ethical issues for pediatric assent in international research, we propose a way forward in approaching pediatric assent for research subjects within the AMPATH collaborative research program in western Kenya that may apply to other multinational research partnerships.

\section{Current State of Pediatric Assent in Kenya}

Although it was a new issue for our research collaboration, consideration of pediatric assent has been documented in other research settings in Kenya. In a qualitative study examining opinions of Kenyan community members related to informed consent for research, Molyneux et al. asked community focus groups whether consent should be sought from Kenyan children from whom blood samples would be requested (Molyneux et al., 2005b). They reported that all of the groups "reacted with surprise" to the question of considering consent for children, with "general agreement that children should not be asked," and they further specified that investigators should not consider asking children aged ten to twelve years (Molyneux et al., 2005b). The study participants reported concerns that the children could not "reason things out" or understand why the samples were needed, and they felt that fear of pain would be the children's only consideration (Molyneux et al., 2005b). Even for children ages ten to thirteen years, the Kenyan community members felt that the researchers should discuss the project directly only with the parents, and then the parents could relay the information to the children (Molyneux et al., 2005b). We could not find any literature documenting how often or in what manner pediatric assent is incorporated in biomedical research in Kenya or in sub-Saharan Africa.

\section{Current State of Pediatric Assent in the United States}

In the United States, research on pediatric assent exists, but this research suggests that pediatric assent is implemented in very different ways from one locality to another. United States federal regulations for the protection of human subjects state that, when the IRB determines that assent is required, the IRB shall determine whether and how assent must be documented (45 CFR $46,404)$. In a study reviewing pediatric assent and consent documents approved by 55 local IRBs in 23 states reviewing three standardized, multicenter clinical trial protocols involving children, Kimberley et al. found that the requirements for assent differed dramatically among the institutions (Kimberly et al., 2006). Working from the same standardized research protocols, 83 percent included some method for documenting child participant assent. Of 69 sets of approved forms, 33 included a line for assent on the informed permission or consent 
form, while 35 required a separate, simplified form for pediatric assent (Kimberly et al., 2006). Thirty-one forms specified an age range at which pediatric assent needed to be secured, but the specified age ranges varied considerably (Kimberly et al., 2006). While some IRBs mandated obtaining pediatric assent from sixyear-olds, others established the age to obtain assent as late as fifteen years (Kimberly et al., 2006). These examples compare with the policy of the IUPUI IRB, which typically requires written pediatric assent be obtained from children seven years and older (IUPUI, 2008).

\section{Professional, National, and International Oversight on Assent and Consent with Pediatric Research Subjects}

The moral foundation for informed consent and assent involving children in U.S. regulations was well described by the National Commission in its report "Research on Children" (National Commission, 1977) and in the Belmont Report (National Commission, 1979). The relevant principle in Belmont is respect for persons:

The principle of respect for persons thus divides into two separate moral requirements: the requirement to acknowledge autonomy and the requirement to protect those with diminished autonomy.

While there is much about Belmont that is applicable to children, there are still areas of disagreement (Childress, Meslin, \& Shapiro, 2005). For example, this principle could be interpreted to support separating children into two categories: older, more mature children with the capacity to be autonomous versus very young children who should be protected and from whom assent is not required (Wendler \& Shah, 2003). Alternatively, others interpret this principle to require that all individuals, even very young children, participate in decision making to the extent that they are able (Nelson \& Reynolds, 2003).

A review of selected guidelines reveals some important variations in the details of how assent, capacity, or age are defined. U.S. regulations for the protection of human subjects include special protections for children (45 CFR 46, Subpart D) which define children as "persons who have not attained the legal age for consent to treatments or procedures involved in the research, under the applicable law of the jurisdiction in which the research will be conducted" and assent as "a child's affirmative agreement to participate in research," noting that "mere failure to object should not, absent affirmative agreement, be construed as assent" (45 CFR $46.406[\mathrm{a}][\mathrm{b}])$. For research not involving greater than minimal risk, federal regulations permit investigators to involve children only if the IRB finds that "adequate provisions are made for soliciting the assent of the children and the permission of their parents or guardians" (45 CFR 46.404). The responsibility of determining when and how pediatric assent will be obtained is left to the IRB (Ungar, Joffe, \& Kodish, 2006); the IRB can determine "whether and how assent must be documented"(45 CFR 46.404 and 408), leaving the process less defined than documenting informed consent in adults. While the regulations do not specify an age at which pediatric assent is recommended, the National Commission recommended obtaining assent for children ages seven years and older, with the objection of a child of any age considered as binding (National Commission, 1977).

The American Academy of Pediatrics (AAP) Committee on Bioethics recommends that pediatric assent should include helping the patient achieve a developmentally appropriate awareness of their condition, telling the patient what to expect, making a clinical assessment of the patient's understanding, and "soliciting an expression of the patient's willingness to accept the proposed care" (Committee on Bioethics, American Academy of Pediatrics, 1995). These guidelines do not distinguish between assent for research and assent for treatment, nor do they define what is "developmentally appropriate" and how exactly the patient's willingness should be solicited or documented. For pediatric participation in studies of drugs, the AAP endorses that seven years be used as the age from which assent for research must be obtained (Committee on Drugs, American Academy of Pediatrics, 1995).

In Kenya, the Guidelines for the Ethical Conduct of Biomedical Research Involving Human Subject published by the Kenya National Council for Science and Technology support respect for the dissent of pediatric research subjects, stating that when "the child refuses to participate in the research, that refusal must be respected unless there's no other medical alternative from which the child could benefit" (Kenya NCST, 2004). While these guidelines do not specifically mandate pediatric assent, the clear emphasis on respecting a child's dissent might suggest a similar position. These guidelines could also be interpreted to indicate willingness to rely on implied assent, assuming that active dissent was not voiced.

Several international guidelines support the requirement for pediatric assent, while continuing to vary in definition and ages included. The guidelines from the Council for International Organisations of Medical Science (CIOMS) require the investigator to ensure that 
"the willing cooperation of the child should be sought, after the child has been informed to the extent that the child's maturity and intelligence permit" and that "a deliberate objection by a child to taking part in research should always be respected even if the parents have given permission, unless the child needs treatment that is not available outside the context of research, the investigational intervention shows promise of therapeutic benefit, and there is no acceptable alternative therapy" (CIOMS, 2002). The CIOMS guidelines do note that the age of legal consent "differs substantially" among countries, and they leave latitude based on local jurisdiction though they generally propose obtaining pediatric assent at twelve or thirteen years of age and beyond (CIOMS, 2002).

The World Medical Association's Declaration of Helsinki, includes a more inclusive statement about the need to procure pediatric assent:

B25. When a potential research subject deemed legally incompetent, such as a minor child, is able to give assent to decisions about participation in research, the physician should seek that assent in addition to the consent of the legally authorized representative (World Medical Association, 2008).

Helsinki thus requires that investigators obtain pediatric assent, but makes no qualifications about how this might vary with age or about the methods to be used.

Guidelines for gathering information from children and adolescents in international settings jointly developed by the Population Council, Family Health International, USAID, and UNICEF recommend obtaining community-level consent from an advisory board that includes children and adults before the activity may proceed (Schenk \& Williamson, 2005). These guidelines also recommend the use of an independent advocate for children to represent the child's views if there is any doubt about whether the guardians have the child's best interests in mind. Other groups, such as UNESCO (UNESCO, 2002) and the OECD (OECD, 2008) also have proposed guidelines that include assent provisions for children, focusing particularly on genetics studies.

\section{Developmental Considerations Relevant to Pediatric Assent}

Despite the ethical rationale for obtaining pediatric assent and the prevalence of statements about pediatric assent in national and international guidelines, implementation of pediatric assent in any context requires careful consideration of a number of practical and theoretical concerns. The details make a difference. For children, several important considerations for pediatric assent are linked to the child's development. The age threshold set for pediatric assent, factors affecting a child's ability to make decisions, and the specific vulnerabilities of pediatric research subjects all merit reflection.

The age at which assent is sought has both practical and theoretical implications. Practically speaking, setting a chronological age at which assent must be obtained allows clear direction for implementing an assent process. However, as we note above, the age thresholds set by IRBs can range widely across institutions and across protocols (Kimberly et al., 2006), and the validity of these age guidelines is open to question. Obtaining assent at seven years of age and older is relatively common, but the primary defense for this position is the "Rule of Sevens" that originated in the 1300s (Wendler, 2006). This rule states that children under the age of seven lack the capacity to make their own decisions, while children from seven to fourteen are presumed not to have this capacity until proven otherwise, and children over fourteen years of age are presumed to have decision-making capacity unless proven otherwise (Wendler, 2006). Wendler has argued that the long history of this "rule" does not necessarily mean it is developmentally appropriate to use to determine pediatric assent practices, nor does the rule even clearly support asking for assent after age seven (Wendler, 2006). Moreover, studies of children's understanding of clinical trials provide evidence that the threshold should be set at nine years or at eleven years (Tait, Voepel-Lewis, \& Malviya, 2003; Ondrusek et al., 1998).

In addition to the crucial concept of age, a number of other factors can influence a child's ability to give assent. First, maturity may be a greater factor in the ability to make decisions and weigh risks than chronological age (Barfield \& Church, 2005; Lewis, 1981). The psychological state of the child, as well as the child's particular medical diagnosis, could impact their feelings of control (Dorn, Susman, \& Fletcher, 1995; Meaux \& Bell, 2001). The child's role and relationships with others and the perceived impact of disagreeing with important figures can impact the assent process (Johnston, 2006; Broome \& Richards, 2003). In addition, factors such as who is asked to obtain assent (Meaux \& Bell, 2001) and whether the child has previous experience with research (Broome \& Richards, 1998) can sway the child's decision of assent or dissent without consideration of the risks and benefits.

For pediatric assent, researchers must also consider the particular vulnerabilities of the pediatric population within research. Kipnis identified seven concepts of 
vulnerability in the pediatric research subject, including children's lack of capacity to make mature decisions, being subject to the authority of others, and being deferential in ways that mask underlying dissent (Kipnis, 2003). While these vulnerabilities in some ways underpin the need to support and protect pediatric assent, they also can impinge on the assent process itself, undermining the extent to which assent provides real protection, and instead creating a sense of false security that children are protected merely because one obtained assent. Assent may not be legitimate if children's capacities, roles, and relationships undermine their ability to provide independent assent.

\section{Cultural Context for Pediatric Assent in Kenya}

Within international research settings, particular threats to informed consent may arise. These threats include significant differences in education between investigators and community members (Shapiro \& Meslin, 2001); mismatches in the understanding, value, and priorities of health and illness (Fairhead, Leach, \& Small, 2006); and limited access to quality health services (Emanuel et al., 2004). While prior studies deal almost exclusively with adult informed consent, pediatric assent is likely to be complicated by the same issues. Furthermore, individual countries are likely to have specific social and cultural factors influencing pediatric assent.

With regard to Kenya, certain social and cultural characteristics define the rights and status of children, shape the perception and understanding of informed consent generally, and determine the view of clinical research. These culturally specific characteristics could impinge on the ability to obtain ethical, legitimate pediatric assent within the Kenyan research setting.

The traditional cultural and social understanding of children in Kenya is unlikely to include a conception of the child as an independent agent. According to the ethnographic literature, people living in indigenous communities in Western Kenya are considered first and foremost as parts of a social group, rather than as independent agents with autonomy to make their own decisions (Dickerson-Putman \& Brown, 1998; Lijembe, 1969; Oboler, 1985; Weisner, 1997; Ember \& Ember, 2003). Even individual health-related behaviors, such as daily medication adherence and child care, are significantly affected by community perceptions and the cultural context (Vreeman et al., 2008). Adults are traditionally given the decision-making role within the predominant ethnic groups in Western Kenya, while the children are expected to show obedience and respect to their elders by carrying out the adults' decisions (Lijembe, 1969; Oboler, 1985). If children do not do as they are told or are not considered respectful, they can expect to be beaten (Pinheiro, 2005). However, this conception of the child may be changing (Weisner, Bradley, \& Kilbride,1997). Anecdotally, members of the Kenyan investigative team and the MU IREC suggested that, with education becoming more prevalent and more children being raised in urban centers, children may be more apt to ask their parents questions regarding their health and to raise concerns. In particular, increases in the education and literacy levels of adults and children alike were thought to be progressively altering the conception of a child into a conception more similar to the "Western" view.

Children in Western Kenya also may be viewed as an important resource or as property (Lijembe, 1969; Oboler, 1985). They are expected to fulfill the responsibilities assigned by their parents, including childcare or farm work (Oboler, 1985). Among the Nandi, it is even common for children to be fostered out temporarily to other family members who may need a child to help around the house or with the herds and farm. Extended families in Western Kenya thus share in the management, care, and support of children in what is termed a shared management family system (Oboler, 1985). Moreover, there are traditional gender differences in the view of and perceived value of children in Western Kenya. Female children may be more likely to be viewed as property (Oboler, 1985, Halperin, 2005).

These dynamics of the family system and the perceived roles of children in Kenya could have important implications for achieving pediatric assent. The traditions of both shared management of the family and group decision making make the concept of individual consent unusual, particularly for children. If there is a lifelong expectation that children will quietly and respectfully comply with adults' decisions, the children may be unlikely to voice meaningful dissent. The routine use of corporal punishment could make children fear physical harm if they do not give the same answer as the adults responsible for them, regardless of what verbal assurances they may receive. The expectation that children will contribute to the economic stability of the family could make children vulnerable to participating in a study because of the expectation of compensation or of benefits beyond those stated in the study (Nyambedha, 2008).

Differences in how and why children of either gender might be valued could lead to further vulnerabilities for one gender or the other. Even if procedures are in place to obtain pediatric assent, a culture of child submissiveness, 
heightened vulnerability in the child's position within the household, and parental expectations could all lead the child to assent despite his or her own objections. For example, an examination of ethical issues for social science research among Kenyan orphans raised concerns that children were consenting to participation in order to show respect to adults and because they associated research with direct assistance for orphans (Nyambedha, 2008). On the other hand, these same vulnerabilities might compel researchers to attempt to guard the children's safety even more carefully, potentially including the use of pediatric assent for such purposes.

Previous research has explored community views on pediatric consent and study participation in Kenya. Of particular relevance is the perception of children by community members as competent decision makers, given the importance of capacity as a criterion for informed consent. A qualitative study by Molyneux et al. suggests that children are viewed by the community as unable to "reason out things" for the purposes of informed consent and "cannot understand" what the risks and benefits of a study might be (Molyneux et al., 2005b). In this context, the child is not seen as a legitimate decision maker, a factor that further undermines their ability to fully assent or dissent. Children are not the only agents perceived as lacking the ability to consent in Kenya; community members also suggested that males should make decisions within the household and that, in some communities, the women should be allotted the same status as children (e.g., not be considered capable of consenting) (Molyneux et al., 2005b).

The informed consent process in Kenya is further complicated by difficulties in distinguishing research from health interventions, participation in research because of expectations of assistance, and the potential need to incorporate verbal consent. Research on informed consent from the east coast in Kenya revealed that consent was based primarily on broad trust in the institution conducting the research and not on the particulars of any particular study protocol (Molyneux, Peshu, \& Marsh, 2005a). Furthermore, potential research subjects may be swayed by the therapeutic misconception in which, despite explanations to the contrary, research participants mistakenly believe that the research project will be of direct benefit to them. While originally described about research in economically developed countries (Appelbaum, Roth, and Lidz, 1982), the therapeutic misconception has been described in other international settings (e.g., Hardy et al., 1998). The therapeutic misconception can be a problem in any setting, but it may be a particular concern in settings with less research literacy and poorer access to health care. With respect to research literacy, prior studies in Kenya have shown that potential research subjects experienced this misconception (Molyneux, Peshu, \& Marsh, 2004a). The subjects also viewed compensation for research participation as an appreciated assistance (Molyneux et al., 2005b). With respect to access to care, the National Bioethics Advisory Commission noted that, in resource-poor countries, where participation in research may be the only time when individuals come in contact with a health care professional, one should be careful about attaching the label of "misconception":

[I]t is not a misconception to believe that participants will probably receive good clinical care during research. But it is a misconception to believe that the purpose of clinical trials is to administer treatment rather than conduct research (NBAC, 2001: 48).

The expectations of aid in return for research has also been highlighted as a concern for social science research within Kenya (Nyambedha, 2008). Moreover, relying on written informed consent may be considered less ethical in Kenya than in economically developed western countries. Our prior experience within the Indiana University-Moi University collaboration stressed the need to implement verbal informed consent (Sidle et al., 2006). Incorporating the already complicated concept of pediatric assent into this informed consent process in Kenya thus requires even greater attention to these issues.

\section{Working Towards Resolution in the Context of a Research Collaboration in Kenya}

Emanuel et al. have developed a framework for ethical clinical research in developing countries that includes collaborative partnership as a key principle for multinational clinical research (Emanuel et al., 2004). Among the benchmarks for this principle is respect for the community's values, culture, traditions, and social practices. Respect for the community's values, culture, and tradition was also highlighted as a key concern for members of both the IUPUI IRB and the MU IREC (Sidle et al., 2006). As highlighted within the case presented, institutional research ethics committees may advocate different requirements for pediatric assent, reflecting both the general, substantial challenges with conceptualizing pediatric assent and specific community values and cultural factors related to the view of children. Nonetheless, a respectful collaborative partnership engages with the involved communities to resolve apparent conflicts and to maximize the ethical conduct of research (Sidle et al., 
2006) In the quest to balance respect for the community's values and culture with national and the protection of and respect for the child intended by pediatric assent, community engagement also presents the way forward.

One step in engaging the involved community is to consult directly with the local institutional research ethics committee. This is consistent with the expectation that the responsibility for implementing a pediatric assent process generally rests with the local IRB (Ungar et al., 2006). In our particular case, we found that members of the MU IREC had widely varying attitudes toward pediatric assent. Some members were reportedly in favor of obtaining pediatric assent and viewed obtaining children's assent as an outgrowth of the Children's Act, which stresses children's rights (Laws of Kenya, CAP 586), or as a concept congruent with international standards. These members felt that implementing pediatric assent practices would be an appropriate step within the current cultural context. Others felt that pediatric assent would not be appropriate in this setting since "our children are brought up to go by their parents' decision." Members disagreed among themselves about the appropriate age cut-off, with some members agreeing that seven years was an appropriate age at which to expect the child to "have cognition" and others feeling an older age would be more appropriate. Some MU IREC members felt that pediatric assent should only apply for children without living biological parents, based on the presumption that parents act in the best interest of their children, whereas orphans may require special safeguards.

\section{Collaboration and Consensus}

Reaching consensus on the implementation of our study procedures also required discussion on the part of our study team of Kenyan and American investigators. Although our team had some hesitations, we had decided to put forward a protocol that would include a component of pediatric assent. Using a template for pediatric assent from the IUPUI IRB, we drafted a version of a pediatric assent form for the study in English, which was subsequently translated into Kiswahili (Figure 2). For children ages seven years and older, we proposed to have the Kiswahili assent form read aloud to them by the study research assistant in the presence of a witness and the research assistant. Children's verbal assent or dissent was then signified on the assent form by having the two witnesses sign the form. We defined dissent as either verbal dissent at the time of this procedure or the child saying at any future point that they did not want to participate in the study.
While you are in the hospital overnight, these are the things we will ask you to do:

- Take your usual medicines.

- Have an IV line fixed in your arm. The nurse or doctor will poke a needle into your arm in order to put the IV into one of your veins. A small tube will stay in the vein in your arm while you are in the hospital.

- From this IV tube, we will take out five small blood samples that will be tested to see how your medicines are working in your body. We will also take one sample of blood that we will save in case we want to use it for another test.

- You will need to drink a small amount of medicine that is mixed with water or juice.

- You will have an exam by a doctor where they examine your body from head to toe-just like the doctor does when you usually come to the clinic.

- You will have your height and weight measured on the scale.

- If you take your medicines by yourself, someone who works for the study will ask you questions about what it is like to take these medicines.

Mukiwa hospitalini usiku kucha, yafuatayo ni mambo ambayo tutakuliza wewe ufanye:

- Kuywa/kumeza dawa zako za kawaida.

- Shindano ya mshipa itawekwa kwa mkono wako. Daktari au muuguzi atadunga shindano kwa mkono wako ili tuweke hii shindano ya mshipa kwa moja ya vena yako. Tyubu ndogo itabaki kwa vena ya mkono wako wakati uko hospitalini.

- Toka kwa tyubu hii, tutachukua sampuli ndogo tano za damu ambazo zitapimwa kuona jinsi dawa zinafanya kazi kwa mwili wako. Tutachukua sampuli ingine moja ya damu ambayo tutahifadhi ikiwa tunataka kutumia kwa kuima kwingine.

- Utahitaji kukunywa kiasi kidogo cha dawa ambayo imechanganishwa na juisi au maji.

- Utachunguzwa na daktari ambaye atachunguza mwili wako kutoka kwa kichwa mpaka kwa kidole cha mguu wako-jinsi vile daktari hufanya unapokuja kliniki.

- Urefu wako na uzito utapimwa kwa ratili.

- Ukikunywa madawa wewe mwenyewe, mtu anayefanya kazi kwa uchunguzi huu atakuuliza maswali kuhusu jinsi ilivyo kukunywa/kumeza madawa haya.

FIG. 2. Excerpt from Pediatric Assent Document in English and in Kiswahili.

Following these discussions, the MU IREC ultimately voted to approve the protocol, including procedures to obtain verbal pediatric assent for children seven years of age and older, with a motion made that pediatric assent needed more discussion. This manuscript was drafted as part of that continued discussion. Despite our decisions for this protocol, several questions remained, and other solutions could have been considered. The use of seven years as a threshold and the ability for a child to assent or dissent validly in this social context continues to be debated among the investigators. Would these concerns undermine the legitimacy of pediatric assent in this particular setting? If so, a different conceptualization 
of pediatric assent, or even waiving pediatric assent could have been considered (and, arguably, defensible within U.S. regulations). Furthermore, is there a need to define more specifically a particular family's view of and treatment the child and to tailor the assent process to that view, or should a universal assent process be adapted for the study regardless of education level, language abilities, or view of the child? Another solution would have been to argue that pediatric assent could be waived due to the prospect of direct benefits to enrolled subjects that were not available outside of the research context (Wendler, 2006). Given that the Kenyan guidelines emphasize respect for a child's refusal, we also question how to define this refusal. Would nonverbal acts of refusal such as crying or unhappiness with a blood draw necessitate the removal of children from the study? And was the Kiswahili translation enough or should we investigate in which language the child thinks and try to assess assent in that language? Continuing to evaluate these issues and this experience remain important priorities as the research collaboration moves forward.

\section{Summary}

We presented a case in which an international research collaboration grappled with implementing pediatric assent for a clinical research project in a new context, specifically within an HIV care system in the lowincome country of Kenya. A careful consideration of this case raises important points for both the specific issue of implementing pediatric assent procedures across cultures and for the broader challenge of reaching consensus on the implementation of ethical procedures within an international research collaboration. We considered the breadth of accepted pediatric assent practices, the various recommendations from bodies of oversight, developmental considerations specific to the vulnerable population of children, and the specific cultural context in which our research would take place. The broader challenges for an international research collaboration remain - to find consensus through honest engagement and discussion.

\section{Best Practices}

Respect for the independence of the institutional ethics review committees requires continued commitment to collaboration and communication, particularly when resolution is needed for a given ethical dilemma. Although it is challenging to specify how such resolution should take place, the process followed in this analysis of pediatric assent provides one potential model of best practices: examining relevant national and international guidelines, considering the local and general ethical challenges, and then engaging in open dialogue with community members, investigators, and institutional ethics review committee members might lead to consensus or other resolution. Moreover, even in the face of an imperfect system for truly informed consent for pediatric research, or perhaps because of these limitations (Kodish, 2003), investigators considering biomedical research involving children must maintain heightened awareness of their responsibility to protect this vulnerable population.

We also recommend four specific steps to move towards best practices in implementing pediatric assent in international research collaborations. First, we urge consideration of obtaining witnessed verbal consent or assent in settings with varying degrees of literacy. Second, study explanations and consent procedures should be carried out, whenever possible, in the primary language of the child and the parents, or every effort should be made to utilize appropriate translators. Third, research collaborations should develop standardized, pretested materials to describe common pediatric study procedures such as blood draws. These materials should be developed and tested for face validity and comprehension among adult and child community members. In our experience, the key components for the materials for pediatric assent were a simple description of what would take place and explanations that the child's assent was sought, but not required. In contrast, the adult informed consent materials focused more on weighing the particulars of the potential risks and benefits involved. However, continued assessment of culturally appropriate materials is highly encouraged. In addition, these materials should be available in the common languages spoken within the clinic population. For verbal consent procedures, these materials should be read aloud to the participants, with ample opportunity for questions and answers. Finally, the age thresholds for which assent and consent procedures will be implemented merit continued discussion and investigation.

\section{Research Agenda}

Our experience highlights several areas in which additional research could increase the capacity to conduct ethical research in children. Specific areas of research inquiry previously mentioned include investigating the face validity and reliability of tools to elicit pediatric assent and caregiver consent, as well as investigation into age-specific comprehension. We also need to 
increase community involvement to assess community understanding of informed consent and of pediatric assent. Community involvement will guide efforts to identify and respond to issues related to informed consent (Gikonyo et al., 2008), but it should go hand-in-hand with in-depth study of the community's view of children's rights and decision-making capacity. Qualitative research methods should be used to ascertain a particular community's beliefs about children and assent. An additional research step might be to evaluate the impact of a U.S. designation of "equivalent protection" given to Moi's institutional guidelines, as has been proposed for other countries (Lavery et al., 2005). This is a regulatory designation that recognizes another institutions' ethics review guidelines and being substantially equivalent to those in the U.S. Having "equivalent status" in our collaboration in Kenya would mean that protocols could be reviewed only by the MU IREC, and not require an additional review by the IUPUI IRB. This would not eliminate the need for careful, deliberate dialogue about challenging issues, but we could evaluate whether this strategy leads to the implementation of more culturally sensitive strategies to meet ethical standards. Another option would be to evaluate the functioning of an "international" collaborating ethics review board that included members from all of the collaborating research partners.

\section{Educational Implications}

This consideration of pediatric assent in an international research collaboration has several educational implications. First, the research program must ensure that investigators from all parts of the collaboration who want to conduct research in children first receive specific training in the ethics of pediatric research. Ideally, this education would occur collaboratively and would enable enhanced understanding of the particular vulnerabilities of pediatric research participants, as well as the culturally specific views related to children. Second, because of the novelty of implementing pediatric assent in this cultural setting and because of the ways that pediatric assent could conflict with the parent's or guardian's usual view of the child, we need specific education for study personnel related to pediatric assent and caregiver consent. This education should emphasize that open-ended questions and probes must accompany the use of consent forms or documents to better evaluate understanding, fears, and relational dynamics within the family-all of which could impinge on the ability of even the older child to provide assent. Finally, we need to champion increased community involvement in order to learn directly how to best respect the rights of children within this particular setting. Better understanding the community's view of children and family units, in addition to understanding the community's conception of informed consent, will enable us to best identify and respond to challenges to protecting the vulnerable pediatric population within the research context.

\section{Acknowledgments}

The authors would like to acknowledge gratefully the generous assistance of Irene Marete, Duncan Ngare, Edwin Were, and Kipruto Kirwa in expounding on the considerations of the MU IREC; of Jeannette DickersonPutman in explaining how the social conception of the Kenyan child might impact pediatric assent; and of Elizabeth Edenberg in the preparation of this manuscript. The views expressed in this article are those of the authors and do not necessarily represent the view of the Indiana University School of Medicine and the Moi University School of Medicine. Dr. Meslin's research was supported in part by a grant from the Fogarty International Center at the National Institutes of Health [R25TW006070]. The content is solely the responsibility of the authors and does not necessarily represent the official views of the Fogarty International Center or the NIH. The research of all of the authors was supported in part by a grant to the USAIDAMPATH Partnership from the United States Agency for International Development as part of the President's Emergency Plan for AIDS Relief (PEPFAR). The authors have no conflicts of interest to disclose.

\section{Author Note}

Address correspondence to: Rachel Vreeman, Children's Health Services Research, Indiana University School of Medicine, 410 W. 10th Street, HITS 1020, Indianapolis, IN 46202. E-MAIL: rvreeman@iupui.edu.

\section{Authors' Biographical Sketches}

Rachel Vreeman is Assistant Professor of Pediatrics in Children's Health Services Research in the Department of Pediatrics at the Indiana University School of Medicine and Co-Field Director for Pediatric Research for the USAID-Academic Model Providing Access to Healthcare (AMPATH) partnership in western Kenya. Dr. Vreeman's research expertise focuses on children's adherence to antiretroviral therapy. As the co-chair of the multinational Kenya Pediatric Research Working 
Group that operates within AMPATH and a faculty scholar within the Indiana Clinical and Translational Science Institute, she is particularly interested in promoting ethical implementation of research within the vulnerable pediatric population in Kenya. She also serves on the curriculum advisory committee for the IU-Moi Academic Research Ethics Partnership. Dr. Vreeman led the development, analysis, and writing of this manuscript.

Winstone Nyandiko is Senior Lecturer in the Department of Child Health and Paediatrics at Moi University School of Medicine in Eldoret, Kenya and Associate Program Manager for the AMPATH partnership, serving as the Co-Director for the AMPATH research network. Dr. Nyandiko is the Pediatrician-InCharge for the AMPATH Pediatric HIV Care Program and the Neonatal Unit of Moi Teaching and Referral Hospital. Dr. Nyandiko's expertise lies in evaluating the clinical management of HIV-exposed and HIV-infected children and implementing research within the Kenyan health care system. Dr. Nyandiko participated in the development and implementation of the clinical research described in this proposal. He facilitated the discussions of pediatric assent among the multinational ethics review boards, and provided extensive comments to assist in revising the final manuscript.

Eric M. Meslin is Director of the Indiana University Center for Bioethics; Associate Dean for Bioethics; Professor of Medicine and of Medical and Molecular Genetics in the Indiana University School of Medicine; and Professor of Philosophy in the School of Liberal Arts. He served as Executive Director of the National Bioethics Advisory Commission and has published extensively on research ethics and health policy. Dr. Meslin previously directed the development of a Memorandum of Understanding (MOU) between the ethics review boards of Indiana University School of Medicine and Moi University School of Medicine, followed by a joint needs assessment to assess barriers to implementing the MOU. He is currently the principal investigator for a new grant from the Fogarty International Center to develop the IU-Moi Academic Research Ethics Partnership, which will feature dual Master's degrees in international research ethics in Indiana and Kenya. Dr. Meslin participated extensively in the development of this analysis, with significant contributions to the writing and revisions of the final manuscript.

\section{References}

Appelbaum, P. S., Roth, L. H., \& Lidz, C. W. (1982). The therapeutic misconception: informed consent in psychiatric research. International Journal of Law and Psychiatry, 5(3-4), 319-329.

BARfield, R. C. \& Church, C. (2005). Informed consent in pediatric clinical trials. Current Opinion in Pediatrics, 17(1), $20-24$.

Broome, M. E. \& Richards, D. J. (1998). Involving children in research. Journal of Child and Family Nursing, 1(1), 3-7.

Broome, M. E. \& Richards, D. J. (2003). The influence of relationships on children's and adolescents' participation in research. Nursing Research, 52(3), 191-197.

Childress, J., Meslin, E., \& Shapiro, H. (Eds.) (2005). Belmont revisited: Ethical principles for research with human subjects. Washington, D.C.: Georgetown University Press.

Committee on Bioethics, American Academy of Pediatrics. (1995). Informed consent, parental permission, and assent in pediatric practice. Pediatrics, 95(2), 314-317.

Committee on Drugs, American Academy of Pediatrics. (1995). Guidelines for the ethical conduct of studies to evaluate drugs in pediatric populations. Pediatrics, 95(2), 286-294.

Council for International Organizations of Medical Sciences, in collaboration with the World Health Organization. (2002). International ethical guidelines for biomedical research involving human subjects. Geneva: Council for International Organizations of Medical Sciences. Available online at http://www.cioms.ch/publications/layout_guide2002.pdf. Dickerson-Putman, J. \& Brown, J. K. (Eds.) (1998). Women among women: Anthropological perspectives on female age hierarchies (pp. 30-51). Champaign, IL: University of Illinois Press.

Dorn, L. D., Susman, E. J., \& Fletcher, J. C. (1995). Informed consent in children and adolescents: Age, maturation and psychological state. Journal of Adolescent Health: Official Publication of the Society of Adolescent Medicine, 16(3), 185-190.

Emanuel, E. J., Wendler, D., Killen, J., \& Grady, C. (2004). What makes clinical research in developing countries ethical? The benchmarks of ethical research. Journal of Infectious Diseases, 189(5), 930-937.

EMber, C. R. \& Ember, M. (Eds.) (2003). Encyclopedia of sex and gender: Men and women in the world's cultures (pp. 247-256). New York: Kluwer Academic Publishers.

Fairhead, J., Leach, M., \& Small, M. (2006). Public engagement with science? Local understandings of a vaccine trial in the Gambia. Journal of Biosocial Science, 38(1), 103-116.

Gikonyo, C., Bejon, P., Marsh, V., \& Molyneux, S. (2008) Taking social relationships seriously: Lessons learned from 
the informed consent practices of a vaccine trial on the Kenyan Coast. Social Science and Medicine, 67(5), 708-720.

HALPERIN, H. (2005). I laugh so I won't cry: Kenya's women tell the story of their lives. Trenton, NJ: Africa World Press.

Hardy, E., Osis, M. J. D., Bento, S. F., Jiménez, A. L., \& Carnevalli, C. R. (1998). Informed consent and Fertility Regulation in Brazil: Final Report. Unpublished. Cited in: National Bioethics Advisory Commission. (2001). Ethical and Policy Issues in International Research: Clinical Trials in Developing Countries. Bethesda, MD: NBAC. Available online at http://www.bioethics.gov/reports/past_commissions/ nbac_international.pdf.

Havens, P. L. \& Gibb, D. M. (2007). Increasing antiretroviral drug access for children with HIV infection. Pediatrics, 119(4), 838-845.

Indiana University-Purdue University Indianapolis (IUPUI). (2008). Standard operating procedures for research involving human participants. Available online at http://www.iupui. $\mathrm{edu} / \sim \mathrm{resgrad} /$ spon/policiescontent.htm.

JOHnston, T. E. (2006). Issues surrounding protection and assent in pediatric research. Pediatric Physical Therapy: The Official Publication of the Section on Pediatrics of the American Physical Therapy Association, 18(2), 133-140.

Kimberly, M. B., Hoehn, K. S., Feudtner, C., Nelson, R. M., \& Schreiner, M. (2006). Variation in standards of research compensation and child assent practices: a comparison of 69 institutional review board-approved informed permission and assent forms for 3 multicenter pediatric clinical trials. Pediatrics, 117(5), 1706-1711.

KIPNIS, K. (2003). Seven vulnerabilities in the pediatric research subject. Theoretical Medicine and Bioethics, 24(2), 107-120.

Kodish, E. (2003). Informed consent for pediatric research: Is it really possible? Journal of Pediatrics, 142(2), 89-90.

Lavery, J. V., McDonald, M., \& Meslin, E. M. (2005). Research ethics across the 49th Parallel: The potential value of pilot testing "equivalent protections" in Canadian research institutions. Health Law Review, 13, 85-96.

Laws of Kenya. (2001). The Children Act. CAP 586.

LEwIS, C. C. (1981). How adolescents approach decisions: Changes over grades seven to twelve and policy implications. Child Development, 52, 538-544.

Lijembe, J. A. (1969). The valley between: A Muluyia's story. In L. K. Fox (ED.), East African childhood (pp. 1-41). Nairobi: Oxford University Press.

Meaux, J. B. \& Bell, P. L. (2001). Balancing recruitment and protection: Children as research subjects. Issues in Comprehensive Pediatric Nursing, 24(4), 241-251.

Molyneux, C. S., Peshu, N., \& Marsh, K. (2004). Understanding of informed consent in a low-income setting: Three case studies from the Kenyan coast. Social Science \& Medicine, 59(12), 2547-2559.
Molyneux, C. S., Peshu, N., \& Marsh, K. (2005a). Trust and informed consent: Insights from community members on the Kenyan coast. Social Science \& Medicine, 61(7), 1463-1473.

Molyneux, C. S., Wassenaar, D. R., Peshu, N., \& Marsh, K. (2005b). "Even if they ask you to stand by a tree all day, you will have to do it (laughter)... !": Community voices on the notion and practice of informed consent for biomedical research in developing countries. Social Science \& Medicine, 61(2), 443-454.

National Bioethics Advisory Commission. (2001). Ethical and policy issues in international research: Clinical trials in developing countries. Bethesda, MD: NBAC. Available online at http://bioethics.georgetown.edu/nbac/clinical/Vol1.pdf.

National Commission for the Protection of Human Subjects of Biomedical and Behavioral Research. (1977). Report and recommendations: Research involving children. Washington, D.C.: U.S. Government Printing Office. Available online at http://www.bioethics.gov/reports/past_commissions/Research_ involving_children.pdf.

National Commission for the Protection of Human Subjects of Biomedical and Behavioral Research. (1979). The Belmont Report: Ethical principles and guidelines for the protection of human subjects of research. Washington, D.C.: U.S. Government Printing Office; (1979). Available online at http://www.hhs.gov/ ohrp/humansubjects/guidance/belmont.htm.

National Council for Science and Technology, Kenya. (2004). Guidelines for ethical conduct of biomedical research involving human subjects in Kenya (NCST No. 45). Nairobi, Kenya: National Council for Science and Technology.

Nelson, R. M. \& Reynolds, W. W. (2003). We should reject passive resignation in favor of requiring the assent of younger children for participation in nonbeneficial research. American Journal of Bioethics, 3(4), 11-13.

Nyambedha, E. O. (2008). Ethical dilemmas of social science research on AIDS and orphanhood in Western Kenya. Social Science \& Medicine, 67(5), 771-779.

Oвоler, R. (1985). Women, power, and economic change. The Nandi of Kenya. Palo Alto, CA: Stanford University Press.

Ondrusek, N., Abramovitch, R., Pencharz, P., \& Koren, G. (1998). Empirical examination of the ability of children to consent to clinical research. Journal of Medical Ethics, 24(3), $158-165$.

Organization for Economic Co-operation and Development. (2008) Guidelines for human biobanks and genetic research databases. Available online at http://www.oecd.org/sti/ biotechnology/hbgrd.

Pinheiro, P. S. (2005). World report on violence against children (pp. 31-42). The United Nations Secretary General's Study on Violence Against Children. Geneva: U.N. Publishing Services. Available online at http://www.unicef.org/violencestudy/ I.\%20World\%20Report\%20on\%20Violence\%20against $\%$ 20Children.pdf. 
Schenk, K., \& Williamson, J. (2005). Ethical approaches to gathering information from children and adolescents in international settings: Guidelines and resources. Washington D.C.: Population Council. Available online at http://www.popcouncil. org/pdfs/horizons/childrenethics.pdf.

Shapiro, H. T. \& Meslin, E. M. (2001). Ethical issues in the design and conduct of clinical trials in developing countries. New England Journal of Medicine, 345(2), 139-142.

Sidle, J., Were, E., Wools-Kaloustian, K., Chuani, C., Salmon, K., Tierney, W., \& Meslin, E. (2006). A needs assessment to build international research ethics capacity at Moi University and Indiana University. Journal of Empirical Research on Human Research Ethics, 1(2), 23-38.

Tait, A. R., Voepel-Lewis, T., \& Malviya, S. (2003). Do they understand? (Part II): Assent of children participating in clinical anesthesia and surgery research. Anesthesiology, 98(3), 609-614.

Ungar, D., Joffe, S., \& Kodish, E. (2006). Children are not small adults: Documentation of assent for research involving children. Journal of Pediatrics, 149(1), S31-S33.

United Nations Children's Fund (UNICEF). (2007). The State of the World's Children 2007. Women and Children: The Double Dividend of Gender Equality. New York: UNICEF. Available online at http://www.unicef.org/sowc07/docs/ sowc07.pdf.

United Nations Educational, Scientific, and Cultural Organization (UNESCO). (2003). International declaration of human genetic data. Available online at: http://portal.unesco.org/
en/ev.php-URL_ID=17720\&URL_DO=DO_TOPIC\&URL_ SECTION=201.html.

U.S. Department of Health and Human Services, National Institutes of Health, and Office for Human Research Protections. (2005). Code of Federal Regulations, Title 45 (Public Welfare), Part 46 (Protection of Human Subjects). Available online at http://www.hhs.gov/ohrp/humansubjects/ guidance/45cfr46.htm.

Vreeman, R., Nyandiko, W., Ayaya, S., Walumbe, E., Marrero, D., \& InUi, T. (2008). Pediatric adherence to antiretroviral therapy among HIV-infected children in Western Kenya: A culturally grounded conceptual model. Paper presented at AIDS 2008 Conference, Mexico City, Mexico.

Weisner, T. S., Bradley, C., \& Kilbride, P. L. (Eds.) (1997). African families and the crisis of social change. Westport, CT: Bergin \& Garvey.

Wendler, D. \& Shah, S. (2003). Should children decide whether they are enrolled in nonbeneficial research? American Journal of Bioethics, 3(4), 1-7.

WENDlER, D. S. (2006). Assent in paediatric research: Theoretical and practical considerations. Journal of Medical Ethics, 32(4), 229-234.

World Medical Association. (2004). Declaration of Helsinki: Ethical principles for medical research involving human subjects. Journal International de Bioethique, 15(1), 124-129.

World Medical Association. (2008). Declaration of Helsinki: Consultation draft. Available online at http://www.wma.net/ e/pdf/doh_review_consultation_draft_may2008.rtf. 